\title{
A Pyroptosis-Related LncRNA signature in Prognosis and Immune Microenvironment infiltration Prediction of Ovarian Cancer
}

Zhao-Yi Liu ${ }^{1}$, Yong-Chang Chen ${ }^{1}$, Nayiyuan $\mathrm{Wu}^{1}, \mathrm{He} \mathrm{Li}^{1,2 \dagger}$, Jing Wang ${ }^{1,3 \dagger}$

${ }^{1}$ Hunan Clinical Research Center in Gynecologic Cancer, Hunan Cancer Hospital and The Affiliated Cancer Hospital of Xiangya School of Medicine, Central South University, 283, Tongzipo Road, Changsha, 410013, Hunan, People's Republic of China

${ }^{2}$ The animal laboratory center, Hunan Cancer Hospital and The Affiliated Cancer Hospital of Xiangya School of Medicine, Central South University, Changsha 410008, Hunan, People's Republic of China

3 Department of Gynecologic Cancer, Hunan Cancer Hospital and The Affiliated Cancer Hospital of Xiangya School of Medicine, Central South University, 283, Tongzipo Road, Changsha, 410013, Hunan, People's Republic of China

${ }^{\dagger}$ Equal contribution

Correspondence to: Jing Wang, email: wangjing0081@hnca.org.cn; He Li, email: lihe@hnca.org.cn

\begin{abstract}
Simple Summary

To evaluate the role of pyroptosis-related lncRNAs (PRLs) in clinical outcome and immue microenviroment infiltration in ovarian cancer, 32 prognostic PRLs were screened and a 7-PRLs related signature with prediction accuracy of overall survival (OS) and immune microenvironment infiltration for ovarian cancer were developed based on expression profiles in TCGA dataset. Patients with high-risk score showed worse outcome, lower infiltration abundance and immunotherapy response. Additionally, lncRNA TYMSOS was proved to serve as a novel tumor biomarker. It might promote cell proliferation, invasion and migration via inhibiting the process of pyroptosis by regulating the expression of GPX4. Our findings provided a
\end{abstract}


comprehensive analysis of PRLs, which is available for prognostic prediction and immune microenvironment infiltration in ovarian cancer.

\begin{abstract}
LncRNA and pyroptosis play important roles in cancer development and tumor immune microenviroment. However, pyroptosis-related lncRNAs (PRLs) in ovarian cancer have not been identified and its impact on prognosis and immune response are not fully understood. In TCGA-RNA-seq cohort ( $\mathrm{n}=377), 32$ prognostic PRLs were screened by using pearson correlation analysis and univariate cox analysis. Subsequently, a 7-PRLs related signature with high prediction accuracy of overall survival (OS) for ovarian cancer were developed. Multivariate Cox regression analysis suggested that it might serve as an independent prognostic indicator and the risk score showed significantly associated with prognosis. Except to clinical outcome, the signature was also associated with tumor immune microenvironment infiltration. Patients with high risk score exhibited lower infiltration abundance of MHC class I cells, Type I IFN response and immunotherapy response. Furthermore, it was revealed that TYMSOS was highly expressed and its high expression was associated with worse OS in ovarian cancer. TYMSOS deletion in ovarian cancer cell lines inhibited the cell proliferation, invasion and migration. TYMSOS might promote tumor progression via inhibiting the process of pyroptosis by regulating the expression of GPX4. In conclusion, the prognostic PRLs-related signature constructed in our study can be served as efficient biomarkers for prognostic prediction and immune microenvironment infiltration in ovarian cancer.
\end{abstract}

Keywords: pyroptosis, ovarian cancer, prognosic, immune microenvironment, signature

\title{
1. Introduction
}

Ovarian cancer (OC) is the third most malignant cancer of all female reproductive systems and patients with OC suffer from an extremely high recurrence and mortality 
rate[1]. In 2020, there were 21,750 new cases and 13,940 deaths of ovarian cancer in the United States, and it was the fifth most prevalent cause of death among all women who died of cancer[2]. Due to the absence of obvious early physical signs, the median age of OC patients at diagnosis is 63 years, and over $70 \%$ of EOC cases are reported to be newly diagnosed at terminal stages with a five-year survival rate of approximately $48 \%[3]$. The standard of therapy for OC is tumor cytoreductive surgery in conjunction with platinum-based chemotherapy. However, the vast majority of patients recur within two years. It indicates that recurrence and drug resistance are the major challenges that need to be addressed[4]. Given the limitations of current OC treatment, new targets for therapy are desired to enhance the clinical outcome of OC. In this light, there is an overwhelming urgency for robust new prognostic models to render targeted therapies more plausible.

At present, multiple evidence have demonstrated that pyroptosis played importanted roles in cancer[5]. Pyroptosis, which is morphologically characterized by swollen cell lysis, rupture of cell membranes and the release of cell contents, is a programmed cell death referring to the Gasdermin family-induced and caused by the inflammasome, and ultimately activating a cascade of enlarged inflammatory responses[6-8]. As is know to us, there are two main forms of pyroptosis: i) the caspase-1 dependent classical pathway; ii) the caspase4/5/11 reliant non-classical pathway. In the non-classical inflammasome pathway, bacterial lipopolysaccharide (LPS) identifies and initiates caspase-4/5/11 cleavage of gasdermin D (GSDMD) and induces pyroptosis. In a typical inflammasome pathway, the inflammasome recruits and combines with ASC (CARD-containing apoptosis-associated spot-like protein), resulting in ASC gathering, which in turn recruits procaspase- 1 and activates caspase-1. caspase- 1 is involved in the cleavage and maturation of pro-IL-18/1b and the cleavage of GSDMD. The C-terminal fragment of GSDMD (GSDMD-CT) remains in the cytoplasm. Meanwhile, the released N-terminal fragment of GSDMD (GSDMD-NT) is released, leading to building a porosity in the plasma membrane. Subsequently, the excretion of IL-18/1b causes the influx of water, cell swelling and permeability lysis. [9-11]. Recent findings demonstrated that the 
pyroptosis-related gasdermins playes an emerging role and might serve as potential new therapeutic targets in various diesases, including autoimmune and inflammatory diseases, infectious diseases, deafness, and cancer[12-16]. In cancer, it is suggested that the process of pyroptosis inhibits tumorigenesis and progression, as well as serves as a pro-inflammatory signal, and establishes a microenvironment suitable for tumor cell growth[17-20]. Yet, the exact function of the pyroptosis was poorly investigated in OC.

Long non-coding RNAs (lncRNAs) are identified as a group of RNA molecules over $200 \mathrm{bp}$ in length that are not translated into proteins. They are reported to participate in various biological processes, such as epigenetic modifications, inheritance stamping, chromatin organization, and protein amendment[21]. Several studies have indicated that lncRNAs might be engaged in ovarian cancer and pyroptosis[22-25]. While, previous studies have primarily focused on the utility of protein-coding genes of pyroptosis, lncRNAs associated with pyroptosis have barely been reported in OC [26-28]. Therefore, the identification of pyroptosis-related lncRNAs (PRLs) is essential for deciphering the underlying motifs of pyroptosis in $\mathrm{OC}$ and investigating new therapeutic targets.

In our study, PRLs were firstly screened by using the pearson correlation analysis. Furthermore, a prognostic signature was constructed based on the PRLs. The prognostic signature significantly predicted the clinical outcome of OC patients in high-risk group and low-risk group with a high diagnostic accuracy. In addition, it was correlated with the immune microenvironment infiltration and immunotherapy response. Finally, we validated the effects of TYMSOS on cell proliferation, invasion and migration in OC cell lines.

\section{Results}

\subsection{Clinical characteristics of the study patients}

The mRNA and LncRNA expression profiles of 377 OC subjects were screened in the TCGA-RNA-seq dataset. All patients were randomly separated into training cohort 
$(n=189)$ and test cohort $(n=188)$ cohorts and there was no difference between the two cohorts in age, Stage, Grade, tumor residual, lymphatic invasion, subdivision and chemotherapy (Table1). The flow chart of this study was presented in Figure 1A.

\subsection{Identification of prognostic PRLs in OC patients}

Firstly, we compared the expression of the 33 PRGs between OC tissuses and normal ovarian tissues using the TCGA datasets and GTEx datasets. The results suggested that 31 among 33 PRGs were either upregulated or downregulated in OC, compared to normal ovarian tissues (Figure 1B). Then, we identified 14826 lncRNAs in the TCGARNA-seq dataset, based on the lncRNA annotation file from the GENCODE website. To extract the potential PRLs, the Pearson correlation analysis was performed to assess the correlation between PRGs and lncRNAs. Subsequently, we obtained 2792 PRLs in the TCGA-RNA-seq dataset (Supplement Table 2). To assess the prognostic value of PRLs, we initially used univariate Cox regression analysis and identified 32 LncRNAs with a significant value $(\mathrm{p}<0.05)$. The forest plot demonstrated that 32 RPLs were notably associated with the prognosis of OC patients (Figure 1C). Besides, the Cytoscape software (3.8.2) was applied to briefly display the interactive relationships between prognosis PRLs and PRGs (Figure 1D).

\subsection{Construction and validation of the prognostic PRLs signature}

To establish an optimal prognostic signature for predicting clinical outcome in OC patients, the LASSO-Cox regression analysis was performed to screen out the most robust model from the candidated lncRNAs (Figure 2A-B). In summary, we constructed a prgnostic PRLs signature and the risk score for each patient was assigned based on the coefficients of each lncRNA in the TCGA cohort (Figure 2C). The following is the formula for calculating the risk score: The risk score $=(0.1506) * \mathrm{RP} 11-443 \mathrm{~B} 7.3+(-$ $0.1965) *$ CELSR3-AS1 + (0.1693) * RP11-213H15.1 + ( -0.1342) * IFNG-AS1 + ($0.3121) * \mathrm{AC} 018647.3+(0.2218) * \mathrm{CTC}-246 \mathrm{~B} 18.8+(-0.3302) * \mathrm{AC} 012360.4$. Patients were divided into high-risk group and low-risk group depending on the best cut-off of risk score. Survival curves were obtained using the Kaplan-Meier analysis and the log- 
rank test was conducted to compare OS between the two groups. Kaplan-Meier survival curves exhibited that the OS of patients with lower risk was substantially longer than the high-risk group both in the training cohort and validation cohorts (test and sum cohort) (Figure 2D-F). The time-dependent ROC curve analysis was carried out and the AUC value demonstrated that the signature harbored a promising ability to predict the 3-year OS and the 5-year OS, and the prognostic values of the signature in two validation cohorts were consistent with the findings in the training cohort (Figure 2GI). The distributions of the risk scoreand survival status were listed in Figure 3A-F. it suggested that the risk score was associated with survival status. Furthermore, the different expressions of all the seven lncRNAs in the high-risk group and the low-risk group were showed in the heatmap. The four protective lncRNAs (AC018647.3, AC012360.4, CELSR3-AS1, and IFNG-AS1) exhibit low expression in the high-risk group, meanwhile, the other three risk lncRNAs (RP11-443B7.3, RP11-213H15.1, and CTC-246B18.8) have high expression in the high-risk group (Figure 3G-I). All these demonstrated that the prognostic PRLs signature might solidly predict the clinical outcome of patients with OC.

\subsection{Stratification analysis of the prognostic PRLs signature}

To better evaluate the prediction ability of the prognostic PRLs signature, the stratification analysis was performed. Compared to patients with high risk, patients with low risk had better OS in patients aged $\leq 55$ and aged $>55$ subgroup (Figure 4A, $\mathrm{p}=0.00014, \mathrm{p}<0.0001$, respectively). Likewise, the prognostic PRLs signature retained its prognostic ability to predict OS for patients in advanced stage subgroup (Figure 4B, $\mathrm{p}<0.0001$ ), early grade subgroup (Figure $4 \mathrm{C}, \mathrm{p}=0.0032$ ), advanced grade subgroup (Figure $4 \mathrm{C}, \mathrm{p}<0.0001$ ), lymphatic invasion subgroup (Figure 4D, $\mathrm{p}=0.048$ ), no lymphatic invasion subgroup (Figure 4D, $\mathrm{p}=0.0026$ ), no macroscopic disease subgroup (Figure 4E, $\mathrm{p}=0.0045$ ), 1-10 $\mathrm{mm}$ tumor residual subgroup (Figure 4E, $\mathrm{p}=0.00022$ ) and $20+\mathrm{mm}$ tumor residual subgroup (Figure $4 \mathrm{E}, \mathrm{p}=0.00044$ ) . Due to the small samples, there was no remarkable difference in OS between high risk patients and low risk patients in early stage subgroup (Figure $4 \mathrm{~B}, \mathrm{p}=0.19$ ) and $11-20 \mathrm{~mm}$ tumor residual 
subgroup (Figure 4E, $\mathrm{p}=0.078$ ). However, high risk patients in both subgroups had the tendency for worse OS, in contrst to low risk patients. Altogether, these results suggest that the prognostic PRLs retained its ability to predict OS in varous subgroups and it could be served as a potential predictor for OC patients.

\subsection{Modeling the prognostic nomogram}

In addition, whether the prognostic PRLs signature was an independent prognostic facter was assessed by using univariate and multivariate Cox regression analysis. As shown in the forest plot, the red color presented the risk factors $(\mathrm{HR}>1)$ and the blue one indicated the protective factors $(\mathrm{HR}<1)$. The results depicted that risk score (HR: 1.555; 95\% CI: 1.391-1.739; p<0.001), age (HR: 1.022; 95\% CI: 1.009-1.035; $<<0.001)$, stage (HR: 1.395; 95\% CI: 1.045-1.863; p=0.024) but not grade (HR: 1.236; 95\% CI: 0.842-1.814; $\mathrm{p}=0.279$ ) were associated with OS of patients (Figure 5A). Multivariate cox analysis further proved that risk score (HR: 1.529; 95\% CI: 1.367-1.709; $\mathrm{p}<0.001$ ), age (HR: 1.020; 95\% CI: 1.008-1.032; p=0.001), stage (HR: 1.401; 95\% CI: $1.044-$ $1.881 ; \mathrm{p}=0.025$ ) but not grade (HR: 1.100; 95\% CI: 0.741-1.631; $\mathrm{p}=0.636$ ) were independent prognostic factors for OC patients (Figure 5B). Thus, Age, FIGO stage and risk score were applied in the construction of a nomogram model (Figure 5C). Calibration plots indicated that the actual vs predicted rates of 3- and 5-year OS showed perfect concordance (Figure 5D). The diagram verified that the nomogram has a reliable and robust ability to predictive the prognosis for OC patients.

\subsection{Identify the biological function of prognostic PRLs signature in OC}

Gene Set Enrichment Analysis (GSEA) was employed to find the key pathways and biological functions that different in the high- and low-risk groups. Firstly, DEGs were screened and $|\log \mathrm{FC}|>2, \mathrm{p}<0.05$ was identified as statistical significance (Figure 6A). GSEA results suggested that the DEGs were mainly enriched in the inflammatory response pathway, p53 pathway, TGF- $\beta$ signaling and TNF $\alpha$ signaling via NF-kB and so on(Figure $6 \mathrm{~B})$. Then, KEGG analysis and GO analysis were conducted and the outcomes displayed that the DEGs were mainly enriched in cell adhesion molecules, 
MAPK signaling pathway, NF-kB signaling pathway, PI3K-AKT signaling pathway, Wnt signaling pathway, Primary immunodeficiency, and plentiful immune-related biological process (Figure 6C-D). Thus, these results suggested the signature may be involved in the above pathways and influence the survival of OC.

\subsection{Association between PRLs signature and immune infiltration}

Considering the aforementioned enrichment analysis and the stronger association between pyroptosis and immune status, we explored the relationships between risk score and immune microenvironment. To explain the immune cell and stromal cell infiltration situation, wecalculated the ESTIMATEscore, ImmuneScore, PurityScore and StromalScore. The correlation analysis implied that the risk score was positively relevant with the ESTIMATEscore (Figure 7A, $\mathrm{r}=0.14, \mathrm{p}=0.006$ ), and StromalScore (Figrue $7 \mathrm{C}, \mathrm{r}=0.20, \mathrm{p}=9.64 \mathrm{e}-05$ ) but negatively correlated with the PurityScore (Figure $7 \mathrm{D}, \mathrm{r}=-0.14, \mathrm{p}=0.005)$. whereas, there was no correlation between risk score and ImmuneScore (Figure $7 \mathrm{~B}, \mathrm{r}=0.215, \mathrm{p}=0.06$ ). After that, the distribution proportion of different subpopulations of adaptive immunity cells and innate immunity cells in highrisk groups and low-risk groups were analyzed by using the ssGSEA method. The distribution proportion of MHC class I cells and Type I IFN response were significantly lower in the high-risk group than the low-risk group (Figure 7E). In addition, the potential response of individual patients to immunotherapy was appraised by using the TIDE algorithm. The results showed that patients with low risk scores were more sensitive to immunotherapy than patients with high risk scores(Figure 8F), which might be associated with higher expression of PD-L1, CTAL4 and LAG3 in low risk group (Figure 7G-I). Taken together, we speculated that patients with high risk score might recruit multiple immune cells and stromal cells and escape immune surveillance.

Comparison of chemotherapy response between patients with different risk scores Thus far, the predominant therapeutics for OC patients were still chemotherapy and different molecular subtypes of OC should direct clinical decisions. Therefore, we contrasted the sensitivity to a variety of anticancer drugs between the high- and lowrisk groups to imply potential treatment modalities (supplement Table 3). The results 
displayed that the IC50s of veliparib and metformin, two food and drug administration (FDA)-approved drugs, were higher in patients with higher risk. which means the shrinkage risk occurs with the growing sensitivity to veliparib and metformin (Figure 8). Unfortunately, there was no significantly difference in IC50 of cisplatin and paclitaxel between two groups (supplement Figure 1). All these evidence indicated that veliparib and metformin might be a good choice for OC patients with low risk score

\subsection{IncRNA TYMSOS promotes cell proliferation, invasion and migration via inhibiting pyroptosis}

Due to the high expressive abundance, TYMSOS were further validated in SKOV3 and A2780 ovarian cacner cell lines. Firstly, we analyzed the different expression of TYMOS in normal ovarian tissues and OC tissues. The results showed that TYMSOS expression was significantly upregulated in tumor tissues, compared to normal ovatian tissues (Figure 9A). In contrast to patients with low expression of TYMSOS, patients with high expression of TYMSOS had worse OS in GSE26913 dataset (Figure 9B). To reveal the role of TYMSOS in OC, we interfered with the endogenous expression of TYMSOS in SKOV3 and A2780 cell lines with siRNA (Figure 9C). CCK8 assay indicated that TYMSOS silence inhibited cell proliferation (Figure 9D-E). In addition, TYMSOS absence apparently reduced the number of migrated and invaded OC cells (Figure 9F-G). It has been reported that GPX4 defect induced pyroptosis and we found that TYMSOS was positively correlated with the expression of GPX4 (Figure 9H, $\mathrm{r}=0.49, \mathrm{p}=1.27 \mathrm{e}-05)$. More importantly, TYMSOS deletion significantly redcued the expression of GPX4 (Figue 9I). All these indicated that lncRNA TYMSOS might promote cell proliferation, invasion and migration via inhibiting pyroptosis.

\section{Discussion}

Ovarian cancer is one of the most common malignancies in the world and associated with a high mortality rate. Consequently, there is of great importance to identify reliable and effective biomarkers for the OC prognosis. In prior research, the lncRNA signatures 
for prognostic prediction have been valicated in many categories of cancers[29], and there is even a database Lnc2Cancer 3.0, which includes comprehensive data on experimentally supported long non-coding RNAs (lncRNAs) and circular RNAs (circRNAs) associated with human cancers[30]. In the same way, based on the differentially expressed lncRNAs and illness etiology, several lncRNA-associated signatures have correspondingly been industrialized to forecast the outcome of OC patients[31,32]. Nevertheless, the mutual interactions of PRLs in prognosis of OC patients remain unclear. In this study, we firstly reported a prognostic PRLs signature, providing a promising strategy for prognosis and immune response in OC patient, which have important clinical implications for guiding individual treatment and improving the effectiveness of the immune response.

Taking advantage of the TAGA and GTEx databases, we compared the expression of 33 PRGs in OC samples and normal ovary tissues by using the R package "limma". Surprisingly, we discovered that all PRGs except two gene (CASP1 and CASP4) were differently expressed in tumor samples. Afterwards, we performed a correlation examination of the 31 PRGs with lncRNAs. Subsequently, 32 lncRNAs assocaited with OS were picked out and defined as prognostic PRLs. Utimately, several prognostic PRLs were identified to play important roles in cancer. For instance, Overexpression of TOPORS-AS1 was supposed to inhibit ovarian cancer cell proliferation and restrain cell aggressive behavior in vivo and in vitro [33]. LINC01281 was confirmed as immune-associate lncRNAs for Predicting Prognosis in Cervical Cancer[34]. In conclusion, it is still unknown whether these lncRNAs are involved in ovarian oncogenesis and development. Subsequently, we performed LASSO-Cox regression to construct a prognostic PRLs signature. The KM plotter analysis and AUC curves suggested that the prognostic PRLs signature might effectively predict the clinical outcome of OC patients. In further, stratification analysis indicated that the prognostic PRLs signature still retained its prognostic ability to predict OS for patients without considering other clinical features. Multivariate cox regression analysis showed that risk score was an independent risk factor for prognosis of OC patients. For better 
clinical applicability of the signature, we created a nomogram.

GSEA analysis was then carried out between the two groups. The outcomes demonstrated that the model had a robust association with cell proliferation and immunity. What's more, GSDME expression was reported to enhance both the number and functions of tumor-infiltrating natural killer and CD8+ T lymphocytes to increase the phagocytosis of tumor cells[35]. Natural killer cells and cytotoxic T lymphocytes kill gasdermin B (GSDMB)-positive cells through pyroptosis[36]. In addition, lncRNAs was also validated to participate in immune pathways through pyroptosis[37]. Therfore, we then analyzed the different distributions of immune function and immune cells between high- and low-risk group. The results indicated that the risk score was associated with immune microoenvironment and immune response. Various immune cells and immune reponse, especially MHC class I cells and Type I IFN response, were differently distributed in the high-risk group and low-risk group. All the evidence reveals that patients with high risk score might recruit multiple immune cells and stromal cells and escape immune surveillance. Also, we conducted a comparison of the sensitivity of 138 common anti-cancer drugs between the high-risk and low-risk groups. It suggested that patients with high risk acquired a large number of drug-resistance in metformin and veliparib, which acted as the first-line Chemotherapy and Maintenance Therapy in OC [38].

Finally, the expressive abundance of several lncRNAs in ovarian cancer cell lines were detected (Data not shown). Due to the high expressive abundance, TYMSOS were selected for further validation. TYMSOS deletion significantly inhibited the cell proliferation, invasion and migration in A2780 and SKOV3 ovarian cancer cell lines. Inhibition of TYMSOS decreased the expression of GPX4. Besides, TYMSOS was highly expressed in ovarian cancer tissues and high expression of TYMSOS was associated with worse OS in GSE26913 dataset. All the evidences proved that overexpression of TYMSOS promoted cell proliferation and aggressive behavior via reducing pyroptosis and it might serve as a novel target to treat 


\section{Materials and Methods}

\subsection{Data acquisition and preprocessing}

We obtained all datasets shown in this study including TCGA and GTEx datasets were publicly accessible and downloaded from the University of California, Santa Cruz (UCSC) Xena website (https://xena.ucsc.edu). The corresponding clinical information of a total of 377 OC patient samples was available from the TCGA-RNA-seq dataset. The distribution of clinical information was presented in table 1. Normal samples were acquired from the GTEx datasets. The E-MTAB-1814 dataset was the downloaded from the ArrayExpress website (https://www.ebi.ac.uk/arrayexpress/). The GSE26913 dataset were downloaded from GEO database (www.ncbi.nlm.nih.gov/geo/). The quality control and gene expression values were normalized by $\log 2(\mathrm{X}+1)$ transformation with R package "limma" and "reshape2". For the gene annotation, we transformed the Ensembl IDs to gene symbols and genes expression values with multiple probes are determined as the average of the individual probes. The whole TCGA data were randomly split into two equal cohorts: training cohort and test cohort, which was also applied to validate as a sum cohort. There was no different in pathological features and treatment. The PRGs (pyroptosis-related genes) were obtained from the previously published literature and MSigDB dataset (www.gseamsigdb.org/gsea/msigdb/)[22,39].

\subsection{Identification of PRLs}

The lncRNA annotation file was acquired from the GENCODE website for the annotation of lncRNAs. Consequently, $14826 \operatorname{lncRNAs}$ were obtained from the TCGA-RNA-Seq cohort[40]. Pearson correlation analysis was used to screen PRLs. Those lncRNAs with $r>0.4$ and $p<0.001$ were considered as the PRLs[41]. To determine the prognostic value of PRLs, we further conducted univariate Cox regression analysis by using the "survival" package, and the hazard ratios (HR) with 95\% confidence intervals (CIs) were examined. $p<0.05$ indicated that pyroptosis-related lncRNAs were 
significantly associated with overall survival (OS) and considered as prognostic PRLs. PRLs with $\mathrm{HR}>1$ were considered to be risk factors, whereas $\mathrm{HR}<1$ were considered to be protective factors.

\subsection{Construction of prognostic PRLs signature}

A risk signature was constructed by performing the LASSO-Cox regression on the prognostic-related lncRNAs using the "glmnet" package[42]. Through 1000 crossvalidation, a panel of genes and their LASSO coefficients were obtained. The risk score for the signature was calculated using the following formula:

$$
\text { Risk score }=\sum_{i=1}^{n} \beta_{n} x_{n}
$$

( $\mathrm{n}$, is the number of the gene; $\beta$, LASSO coefficient; $\mathrm{X}$, the expression of each prognosticPRLs in each sample). Based on the best cut-off risk score determined by the "surv_cutpoint" function of the "survminer" R package, patients were divided into high-risk and low-risk groups. Kaplan-Meier method with the long-rank test was performed to reveal the difference of OS between the high-risk and low-risk group by using the "survival" package. Besides, the time-dependent ROC curve and area under the curve (AUC) were applied to evaluate the prediction accuracy of the signature. All the time-dependent ROC curves were calculated by the "SurvivalROC" package and drawn by the "ggplot2" package.

\subsection{Nomogram construction based on clinical features and risk score}

Univariate and multivariate COX regression were performed to select the prognostic risk factors. The nomogram model was constructed using the "RMS" package to predict the 3,5-year survival probability. The calibration curves were used to assess the concordance of the observed and predicted rates of 3, 5-year overall survival[43].

\subsection{Estimation of tumor-infiltration, immunotherapy and chemotherapy response}

Firstly, All microenvironment scores, including Estimate score, Immune score, Purity score, and stromal score were calculated by using the ESTIMATE algorithm 
(https://bioinformatics.mdanderson.org/public-software/estimate/)[44]. The infiltrating immune cells scores and the activity of immune-related pathways were calculated by performing the ssGSEA analysis with "gsva" package[45]. Tumor Immune Disfunction and Exclusion (TIDE) algorithm (http://tide.dfci.harvard.edu/), which is commonly utilized to accurately predict the outcome of patients treated with immune checkpoint blockade (ICB), were employed to evaluate the immunotherapy response[46]. The chemotherapy response of each patient was evaluated by using the Genomics of Drug Sensitivity in Cancer database (GDSC, https://www.cancerrxgene.org). The halfmaximal inhibitory concentration (IC50) of all drugs commonly used to treat tumors was calculated and represented the drug response. The R package 'pRRopheticRredic' was used with tenfold cross-validation and other parameters by default[47].

\subsection{Function Enrichment Analysis}

Gene set enrichment analysis (GSEA) was performed to identify the potential molecular mechanisms or potential functional pathways associated with prognostic PRLs signature. The TCGA samples were divided into a high-risk group and a low-risk group according to the optimal cutoff values. GSEA was performed in java GSEA v. 4.0.3 on the molecular signature dataset, h.all.v7.4 symbols.GMT [Hallmarks] and Kyoto Encyclopedia of Genes and Genomes (KEGG) dataset, c2.cp.kegg.v7.4.symbols.GMT. Gene Ontology (GO) enrichment analyses were performed to identify enriched pathways between the high-risk group and the low-risk group by using the "clusterProfiler" R package. $|\mathrm{NES}|>1$ and false discovery rate (FDR) $<0.05$ were considered statistically significant

\subsection{Cell Culture}

The human ovarian cancer cell lines A2780 and SKOV3 were both cultured in RMPI1640 medium, supplemented with $10 \%$ fetal bovine serum (FBS) and $1 \%$ penicillin/streptomycin. They were cultured in a sterile incubator maintained at $37^{\circ} \mathrm{C}$ with $5 \% \mathrm{CO}_{2}$. The cells in logarithmic growth phase were collected for subsequent 
experiments.

\subsection{RNA Extraction and Quantitative PCR}

Total RNA was extracted using TRIZOL reagent according to the manufacturer's protocol (Invitrogen, 15596-026). Reverse transcription of cDNA was performed using the PrimeScript RT kit (Takara, RR047A, Japan). qPCR assays were performed using LightCycler480 detector (Roche, USA). The relative mRNA levels were calculated using the comparative $\mathrm{Ct}$ method with GAPDH as the reference gene. All the primers are listed in supplement Table 1.

\subsection{Colony Forming Assay}

The A2780 and SKOV3 cells were transfected with or without lncRNA-targeted siRNAs for about $48 \mathrm{~h}$. Then, 200 cells were plated in the six-well culture plates and cultured for about 2 weeks. The cellular colonies were counted by staining with crystal violet.

\subsection{Cell Counting Kit-8 assay}

The A2780 and SKOV3 cells were measured for cell survival after transfection with or without lncRNA-targeted siRNAs for $24 \mathrm{~h}, 48 \mathrm{~h}$ and $72 \mathrm{~h}$, respectively. Following the protocols of the manufacturer, cell viability was detected by using Cell Counting Kit- 8 (Proteintech: PF00004).

\subsection{Transwell migration and invasion}

A total of $2 * 10^{5}$ ovarian cells transfected as above were seeded in the upper chamber of Transwell plates with serum-free medium. The cells were then incubated for 48 hours. To perform invasion experiments, the upper chambers were covered with a mixture of RPIM-1640 and Matrigel. Finally, the cells at the top of the lumen were removed with a cotton swab, whereas the cells across the membrane were stained with $0.5 \%$ crystal violet, observed and counted under 100x magnification. 


\subsection{Statistical analysis}

Significant quantitative differences between groups were analyzed using the two-tailed Students' t-test, whereas differences among groups were analyzed using the one-way ANOVA. Kaplan-Meier curves and log-rank test were used to calculate the overall survival rate. All statistical analyses were performed using R sofware (version 4.0.2). * means $p<0.05$, ** means $p<0.01, * * *$ means $p<0.001 . p<0.05$ was considered statistically significant.

\section{Conclusions}

In conclusion, our study constructed for the first time a novel prognostic signature based on lncRNAs associated with pyroptosis. It effectively predicted the clinical out of OC patients. Except to prognosis, the tumor immue microenvironment and immune response were significantly different in high- risk and low-risk group.

\section{Data availability statement}

Publicly available datasets were analyzed in this study. The datasets analyzed for this study were obtained from TCGA, GEO and GTEx databases.

\section{Conflicts of Interest}

The authors declare that there are no conflicts of interest.

\section{Author Contributions}

L.Z.Y contribute to the data collection and analysis. L.Z.Y and L.H wrote the manuscript. W.J designed the study. All authors revised and approved the final manuscript.

\section{Fundings}

This research was supported by the National Natural Science Foundation of China (NO.81972836), National Key R\&D Program (2016YFC1303703) and the Science and 
Technology Innovation Program of Hunan Province (2020RC2065), the Youth Natural Science Foundation of Hunan Province (2021JJ40321)

\section{References}

1. Menon, U.; Karpinskyj, C.; Gentry-Maharaj, A. Ovarian Cancer Prevention and Screening. Obstet Gyneco/ 2018, 131, 909-927, doi:10.1097/AOG.0000000000002580.

2. Siegel, R.L.; Miller, K.D.; Jemal, A. Cancer statistics, 2020. CA Cancer J Clin 2020, 70, 7-30, doi:10.3322/caac.21590.

3. Kuroki, L.; Guntupalli, S.R. Treatment of epithelial ovarian cancer. BMJ 2020, 371, m3773, doi:10.1136/bmj.m3773.

4. Holmes, D. The problem with platinum. Nature 2015, 527, S218-219, doi:10.1038/527S218a.

5. Koren, E.; Fuchs, Y. Modes of Regulated Cell Death in Cancer. Cancer Discov 2021, 11 , 245-265, doi:10.1158/2159-8290.CD-20-0789.

6. Bergsbaken, T.; Fink, S.L.; Cookson, B.T. Pyroptosis: host cell death and inflammation. Nat Rev Microbio/ 2009, 7, 99-109, doi:10.1038/nrmicro2070.

7. Kovacs, S.B.; Miao, E.A. Gasdermins: Effectors of Pyroptosis. Trends Cel/ Bio/2017, 27, 673684, doi:10.1016/j.tcb.2017.05.005.

8. Shi, J.; Gao, W.; Shao, F. Pyroptosis: Gasdermin-Mediated Programmed Necrotic Cell Death. Trends Biochem Sci 2017, 42, 245-254, doi:10.1016/j.tibs.2016.10.004.

9. Miao, E.A.; Rajan, J.V.; Aderem, A. Caspase-1-induced pyroptotic cell death. Immunol Rev 2011, 243, 206-214, doi:10.1111/j.1600-065X.2011.01044.x.

10. Broz, P.; Pelegrin, P.; Shao, F. The gasdermins, a protein family executing cell death and inflammation. Nat Rev Immuno/ 2020, 20, 143-157, doi:10.1038/s41577-019-0228-2.

11. Robinson, N.; Ganesan, R.; Hegedus, C.; Kovacs, K.; Kufer, T.A.; Virag, L. Programmed necrotic cell death of macrophages: Focus on pyroptosis, necroptosis, and parthanatos. Redox Bio/2019, 26, 101239, doi:10.1016/j.redox.2019.101239.

12. Zhu, Q.; Zheng, M.; Balakrishnan, A.; Karki, R.; Kanneganti, T.D. Gasdermin D Promotes AlM2 Inflammasome Activation and Is Required for Host Protection against Francisella novicida. J Immuno/ 2018, 201, 3662-3668, doi:10.4049/jimmunol.1800788.

13. Thurston, T.L.; Matthews, S.A.; Jennings, E.; Alix, E.; Shao, F.; Shenoy, A.R.; Birrell, M.A.; Holden, D.W. Growth inhibition of cytosolic Salmonella by caspase-1 and caspase-11 precedes host cell death. Nat Commun 2016, 7, 13292, doi:10.1038/ncomms13292.

14. Cerqueira, D.M.; Gomes, M.T.R.; Silva, A.L.N.; Rungue, M.; Assis, N.R.G.; Guimaraes, E.S.; Morais, S.B.; Broz, P.; Zamboni, D.S.; Oliveira, S.C. Guanylate-binding protein 5 licenses caspase-11 for Gasdermin-D mediated host resistance to Brucella abortus infection. PLOS Pathog 2018, 14, e1007519, doi:10.1371/journal.ppat.1007519.

15. Xiao, J.; Wang, C.; Yao, J.C.; Alippe, Y.; Xu, C.; Kress, D.; Civitelli, R.; Abu-Amer, Y.; Kanneganti, T.D.; Link, D.C., et al. Gasdermin D mediates the pathogenesis of neonatalonset multisystem inflammatory disease in mice. PLOS Biol 2018, 16, e3000047, doi:10.1371/journal.pbio.3000047.

16. Kanneganti, A.; Malireddi, R.K.S.; Saavedra, P.H.V.; Vande Walle, L.; Van Gorp, H.; Kambara, 
H.; Tillman, H.; Vogel, P.; Luo, H.R.; Xavier, R.J., et al. GSDMD is critical for autoinflammatory pathology in a mouse model of Familial Mediterranean Fever. J Exp Med 2018, 215, 1519 1529, doi:10.1084/jem.20172060.

17. Grivennikov, S.I.; Greten, F.R.; Karin, M. Immunity, inflammation, and cancer. Cel/ 2010, 140, 883-899, doi:10.1016/j.cell.2010.01.025.

18. Molina-Crespo, A.; Cadete, A.; Sarrio, D.; Gamez-Chiachio, M.; Martinez, L.; Chao, K.; Olivera, A.; Gonella, A.; Diaz, E.; Palacios, J., et al. Intracellular Delivery of an Antibody Targeting Gasdermin-B Reduces HER2 Breast Cancer Aggressiveness. Clin Cancer Res 2019, 25, 4846-4858, doi:10.1158/1078-0432.CCR-18-2381.

19. Wang, W.J.; Chen, D.; Jiang, M.Z.; Xu, B.; Li, X.W.; Chu, Y.; Zhang, Y.J.; Mao, R.; Liang, J.; Fan, D.M. Downregulation of gasdermin $D$ promotes gastric cancer proliferation by regulating cell cycle-related proteins. J Dig Dis 2018, 19, 74-83, doi:10.1111/17512980.12576.

20. Liu, X.; Xia, S.; Zhang, Z;; Wu, H.; Lieberman, J. Channelling inflammation: gasdermins in physiology and disease. Nat Rev Drug Discov 2021, 20, 384-405, doi:10.1038/s41573021-00154-z.

21. Statello, L.; Guo, C.J.; Chen, L.L.; Huarte, M. Gene regulation by long non-coding RNAs and its biological functions. Nat Rev Mol Cel/ Bio/2021, 22, 96-118, doi:10.1038/s41580020-00315-9.

22. Ye, Y.; Dai, Q.; Qi, H. A novel defined pyroptosis-related gene signature for predicting the prognosis of ovarian cancer. Cell Death Discov 2021, 7, 71, doi:10.1038/s41420-02100451-X.

23. Li, X.Y.; Zhang, L.Y.; Li, X.Y.; Yang, X.T.; Su, L.X. A Pyroptosis-Related Gene Signature for Predicting Survival in Glioblastoma. Front Oncol 2021, 11, 697198, doi:10.3389/fonc.2021.697198.

24. Shao, W.; Yang, Z.; Fu, Y.; Zheng, L.; Liu, F.; Chai, L.; Jia, J. The Pyroptosis-Related Signature Predicts Prognosis and Indicates Immune Microenvironment Infiltration in Gastric Cancer. Front Cell Dev Bio/ 2021, 9, 676485, doi:10.3389/fcell.2021.676485.

25. Dong, Z.; Bian, L.; Wang, M.; Wang, L.; Wang, Y. Identification of a Pyroptosis-Related Gene Signature for Prediction of Overall Survival in Lung Adenocarcinoma. J Onco/ 2021, 2021, 6365459, doi:10.1155/2021/6365459.

26. Liang, H.; Yu, T.; Han, Y.; Jiang, H.; Wang, C.; You, T.; Zhao, X.; Shan, H.; Yang, R.; Yang, L., et al. LncRNA PTAR promotes EMT and invasion-metastasis in serous ovarian cancer by competitively binding miR-101-3p to regulate ZEB1 expression. Mol Cancer 2018, 17, 119, doi:10.1186/s12943-018-0870-5.

27. Lou, W.; Ding, B.; Zhong, G.; Du, C.; Fan, W.; Fu, P. Dysregulation of pseudogene/IncRNAhsa-miR-363-3p-SPOCK2 pathway fuels stage progression of ovarian cancer. Aging (Albany NY) 2019, 11, 11416-11439, doi:10.18632/aging.102538.

28. Zhao, H.; Ding, F.; Zheng, G. LncRNA TMPO-AS1 promotes LCN2 transcriptional activity and exerts oncogenic functions in ovarian cancer. FASEB J 2020, 34, 11382-11394, doi:10.1096/fj.201902683R.

29. Tan, Y.T.; Lin, J.F.; Li, T.; Li, J.J.; Xu, R.H.; Ju, H.Q. LncRNA-mediated posttranslational modifications and reprogramming of energy metabolism in cancer. Cancer Commun (Lond) 2021, 41, 109-120, doi:10.1002/cac2.12108. 
30. Gao, Y.; Shang, S.; Guo, S.; Li, X.; Zhou, H.; Liu, H.; Sun, Y.; Wang, J.; Wang, P.; Zhi, H., et al. Lnc2Cancer 3.0: an updated resource for experimentally supported IncRNA/circRNA cancer associations and web tools based on RNA-seq and scRNA-seq data. Nucleic Acids Res 2021, 49, D1251-D1258, doi:10.1093/nar/gkaa1006.

31. Yang, H.; Gao, L.; Zhang, M.; Ning, N.; Wang, Y.; Wu, D.; Li, X. Identification and Analysis of An Epigenetically Regulated Five-IncRNA Signature Associated With Outcome and Chemotherapy Response in Ovarian Cancer. Front Cell Dev Biol 2021, 9, 644940, doi:10.3389/fcell.2021.644940.

32. Zheng, M.; Hu, Y.; Gou, R.; Nie, X.; Li, X.; Liu, J.; Lin, B. Identification three LncRNA prognostic signature of ovarian cancer based on genome-wide copy number variation. Biomed Pharmacother 2020, 124, 109810, doi:10.1016/j.biopha.2019.109810.

33. Fu, Y.; Katsaros, D.; Biglia, N.; Wang, Z.; Pagano, I.; Tius, M.; Tiirikainen, M.; Rosser, C.; Yang, $\mathrm{H}$.; Yu, H. Vitamin D receptor upregulates IncRNA TOPORS-AS1 which inhibits the Wnt/beta-catenin pathway and associates with favorable prognosis of ovarian cancer. Sci Rep 2021, 11, 7484, doi:10.1038/s41598-021-86923-7.

34. Ye, J.; Chen, X.; Lu, W. Identification and Experimental Validation of Immune-Associate IncRNAs for Predicting Prognosis in Cervical Cancer. Onco Targets Ther 2021, 14, 47214734, doi:10.2147/OTT.S322998.

35. Zhang, Z.; Zhang, Y.; Xia, S.; Kong, Q.; Li, S.; Liu, X.; Junqueira, C.; Meza-Sosa, K.F.; Mok, T.M.Y.; Ansara, J., et al. Gasdermin E suppresses tumour growth by activating anti-tumour immunity. Nature 2020, 579, 415-420, doi:10.1038/s41586-020-2071-9.

36. Zhou, Z.; He, H.; Wang, K.; Shi, X.; Wang, Y.; Su, Y.; Wang, Y.; Li, D.; Liu, W.; Zhang, Y., et al. Granzyme A from cytotoxic lymphocytes cleaves GSDMB to trigger pyroptosis in target cells. Science 2020, 368, doi:10.1126/science.aaz7548.

37. Wan, P.; Su, W.; Zhang, Y.; Li, Z.; Deng, C.; Li, J.; Jiang, N.; Huang, S.; Long, E.; Zhuo, Y. LncRNA $\mathrm{H} 19$ initiates microglial pyroptosis and neuronal death in retinal ischemia/reperfusion injury. Cell Death Differ 2020, 27, 176-191, doi:10.1038/s41418019-0351-4.

38. Coleman, R.L.; Fleming, G.F.; Brady, M.F.; Swisher, E.M.; Steffensen, K.D.; Friedlander, M.; Okamoto, A.; Moore, K.N.; Efrat Ben-Baruch, N.; Werner, T.L., et al. Veliparib with FirstLine Chemotherapy and as Maintenance Therapy in Ovarian Cancer. N Engl J Med 2019, 381, 2403-2415, doi:10.1056/NEJMoa1909707.

39. Latz, E.; Xiao, T.S.; Stutz, A. Activation and regulation of the inflammasomes. Nat Rev Immuno/2013, 13, 397-411, doi:10.1038/nri3452.

40. Zhang, X.; Sun, S.; Pu, J.K.; Tsang, A.C.; Lee, D.; Man, V.O.; Lui, W.M.; Wong, S.T.; Leung, G.K. Long non-coding RNA expression profiles predict clinical phenotypes in glioma. Neurobiol Dis 2012, 48, 1-8, doi:10.1016/j.nbd.2012.06.004.

41. Hong, W.; Liang, L.; Gu, Y.; Qi, Z.; Qiu, H.; Yang, X.; Zeng, W.; Ma, L.; Xie, J. Immune-Related IncRNA to Construct Novel Signature and Predict the Immune Landscape of Human Hepatocellular Carcinoma. Molecular therapy. Nucleic acids 2020, 22, 937-947, doi:10.1016/j.omtn.2020.10.002.

42. Goeman, J.J. L1 penalized estimation in the Cox proportional hazards model. Biometrical journal. Biometrische Zeitschrift 2010, 52, 70-84, doi:10.1002/bimj.200900028.

43. Li, H.; Wu, N.; Liu, Z.Y.; Chen, Y.C.; Cheng, Q.; Wang, J. Development of a novel 
transcription factors-related prognostic signature for serous ovarian cancer. Scientific reports 2021, 11, 7207, doi:10.1038/s41598-021-86294-z.

44. Yoshihara, K.; Shahmoradgoli, M.; Martínez, E.; Vegesna, R.; Kim, H.; Torres-Garcia, W.; Treviño, V.; Shen, H.; Laird, P.W.; Levine, D.A., et al. Inferring tumour purity and stromal and immune cell admixture from expression data. Nature communications $\mathbf{2 0 1 3}, 4,2612$, doi:10.1038/ncomms3612.

45. Newman, A.M.; Liu, C.L.; Green, M.R.; Gentles, A.J;; Feng, W.; Xu, Y.; Hoang, C.D.; Diehn, M.; Alizadeh, A.A. Robust enumeration of cell subsets from tissue expression profiles. Nat Methods 2015, 12, 453-457, doi:10.1038/nmeth.3337.

46. Jiang, P.; Gu, S.; Pan, D.; Fu, J.; Sahu, A.; Hu, X.; Li, Z.; Traugh, N.; Bu, X.; Li, B., et al. Signatures of $T$ cell dysfunction and exclusion predict cancer immunotherapy response. Nature medicine 2018, 24, 1550-1558, doi:10.1038/s41591-018-0136-1.

47. Nick, T.G.; Hardin, J.M. Regression modeling strategies: an illustrative case study from medical rehabilitation outcomes research. The American journal of occupational therapy : official publication of the American Occupational Therapy Association 1999, 53, 459-470, doi:10.5014/ajot.53.5.459. 


\section{Figure legends}

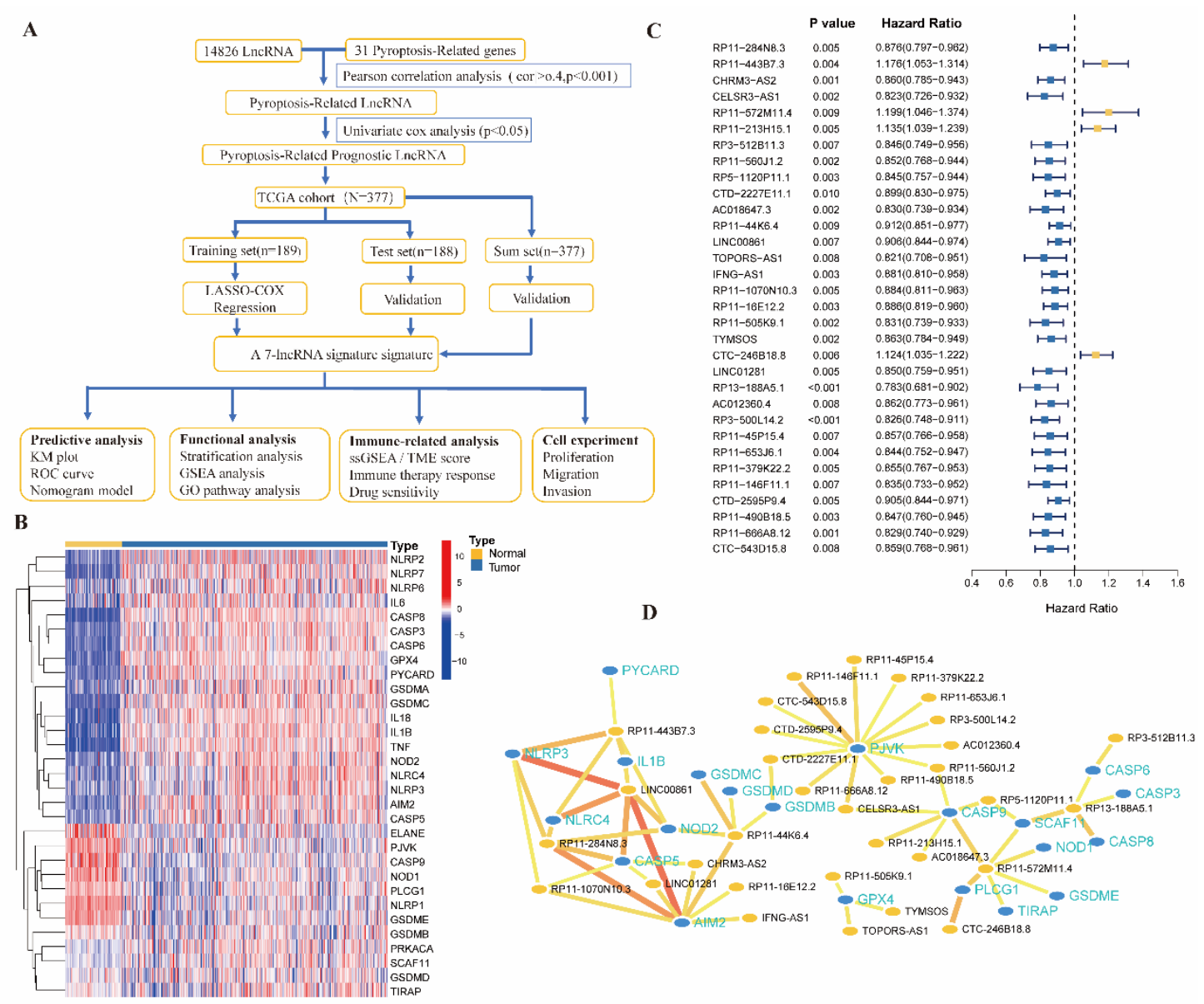

Figure 1. Identification of 31 pyroptosis-related genes and pyroptosis-related lncRNAs; (A) The flowchart of the whole process of data analysis; (B) A Heatmap of the pyroptosis-related genes between the normal ovary and tumor tissues (blue: low expression level; red: high expression level; N: brilliant blue; T, yellow); (C) A forest plot of the prognostic ability of the pyroptosis-related lncRNAs; (D) The interaction network of the prognostic-PRLs and the PRLs (blue: PRGs; orange: prognostic-PRLs). 

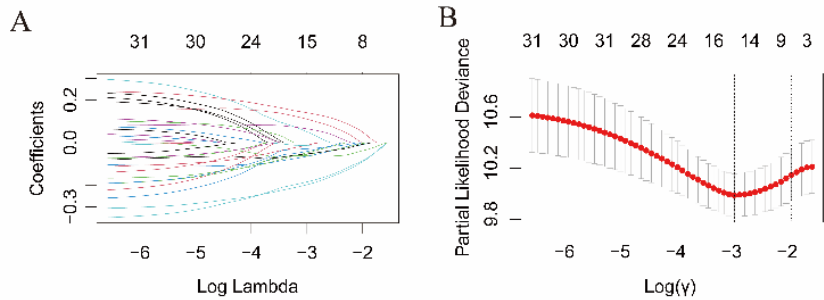

D

Training cohort
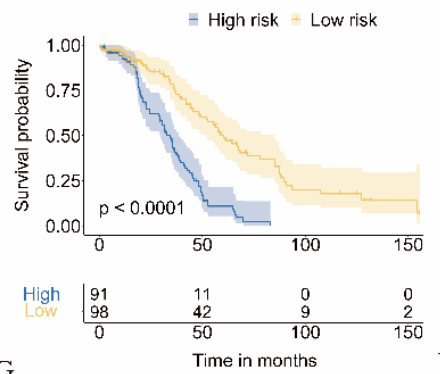

G

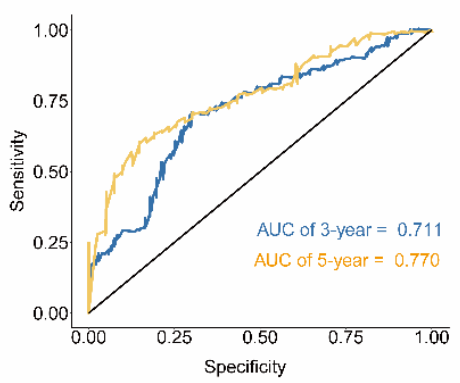

E
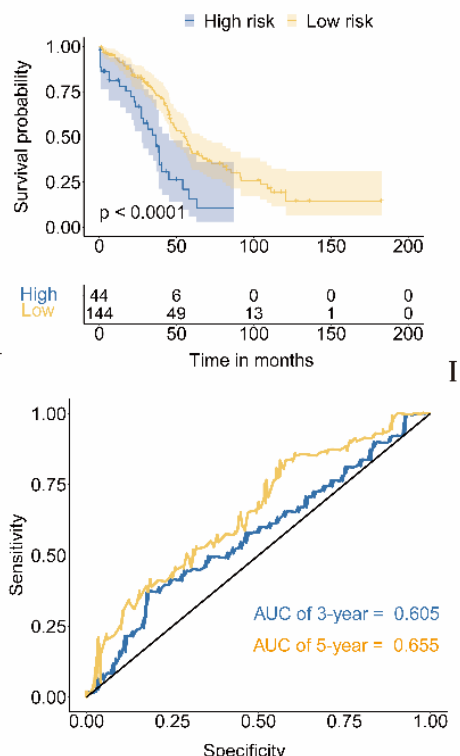

C RP11-443B7.3

RP11-213H15.1

IFNG-AS1

CTC-246B18.8

CELSR3-AS1

AC018647.3

AC012360.4

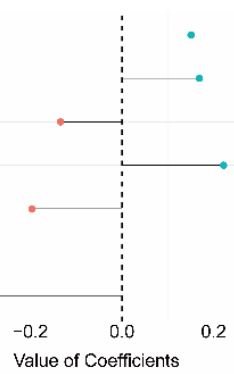

Sum cohort
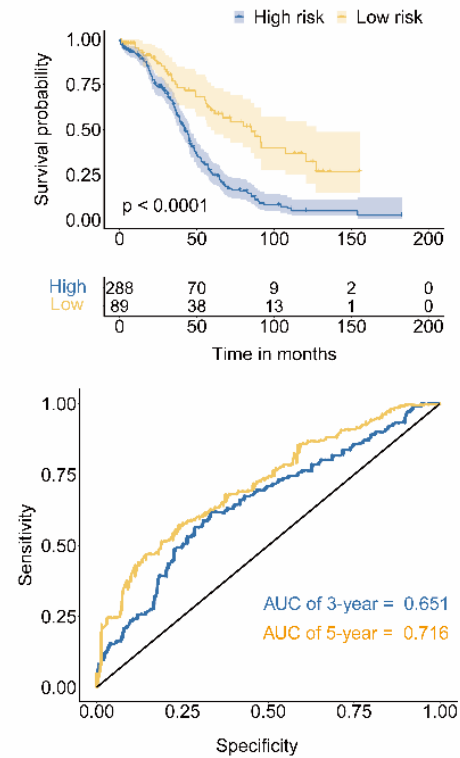

Figure 2. The construction and validation of the prognostic PRLs signature. (A) LASSO regression of the prognostic-PRLs; (B) 10 times cross-validation for tuning the parameter selection in the LASSO regression; (C) The coefficients of the signature; (DF) Kaplan-Meier curves for the OS of patients in the training cohort and the test cohort and the sum cohort; (G-I) The time-dependent ROC curves confirmed the prognostic capabilities of the risk score in the training cohort and the test cohort and the sum cohort. 

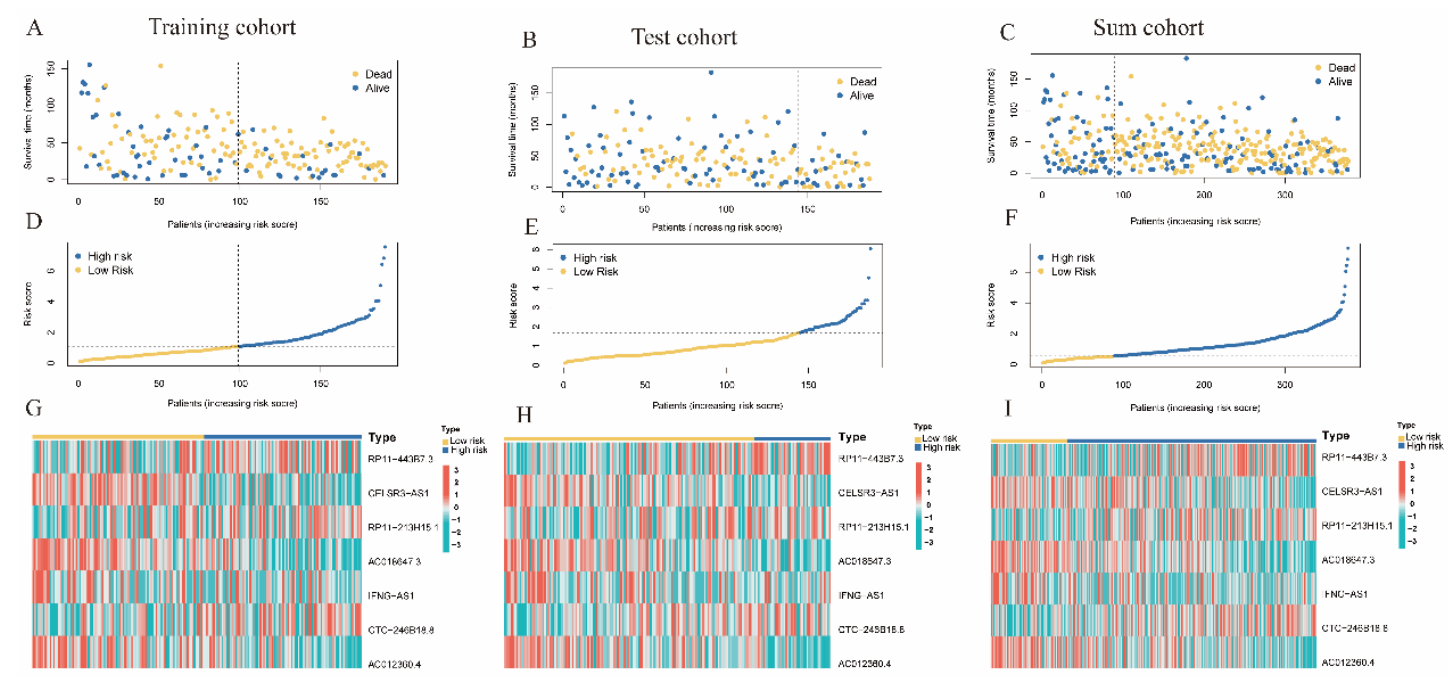

Figure 3. The survival status and gene expression was associated the risk score; (A-C) The Distributions of survival status of OC patients in the training cohort and the test cohort and the sum cohort; (D-F) The risk score calculated by the model in the training cohort and the test cohort and the sum cohort; (G-I) The Heatmap showed the expression profiles of 7 PRLs between the high-risk group and the low-risk group.
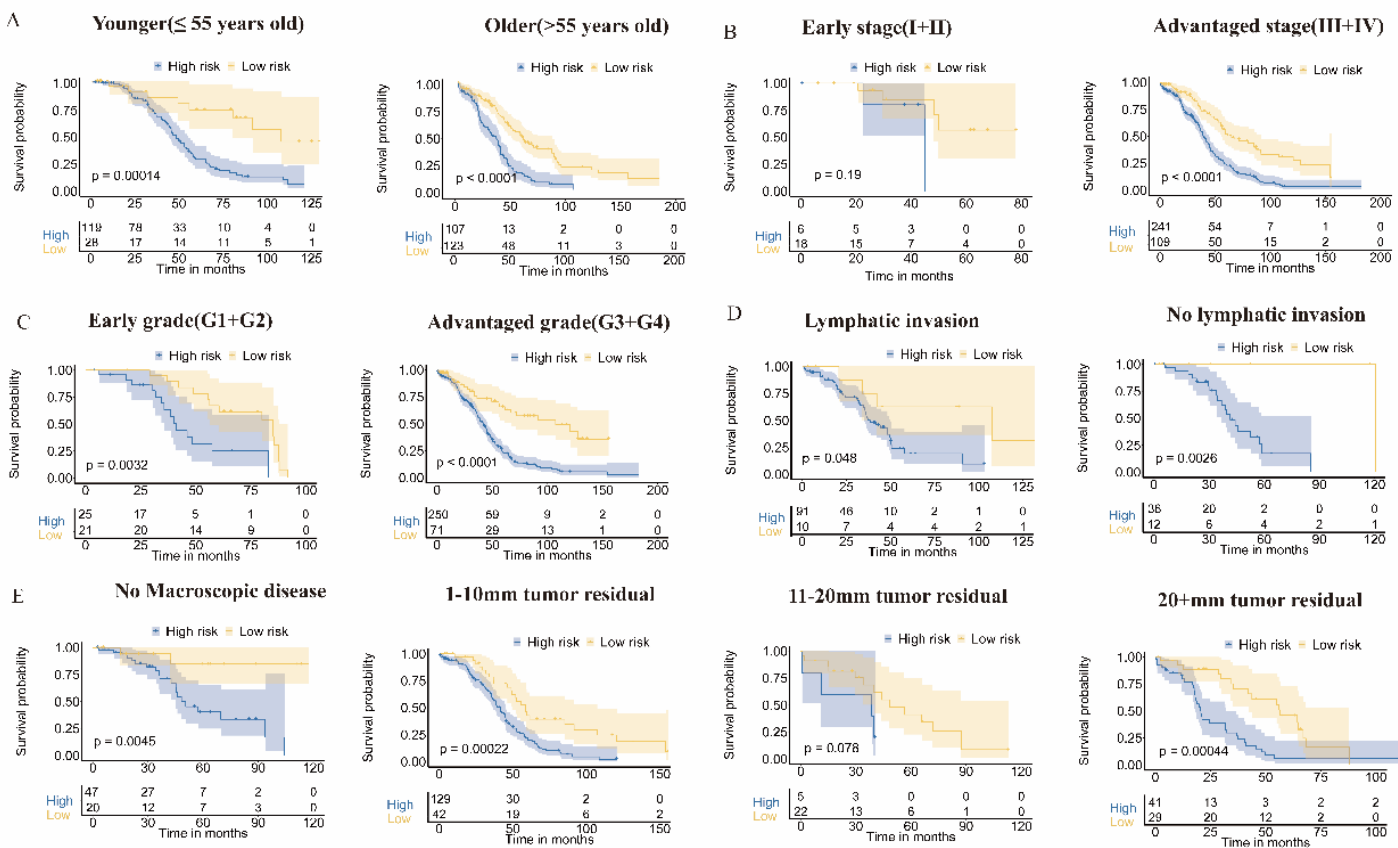

1-10mm tumor residual
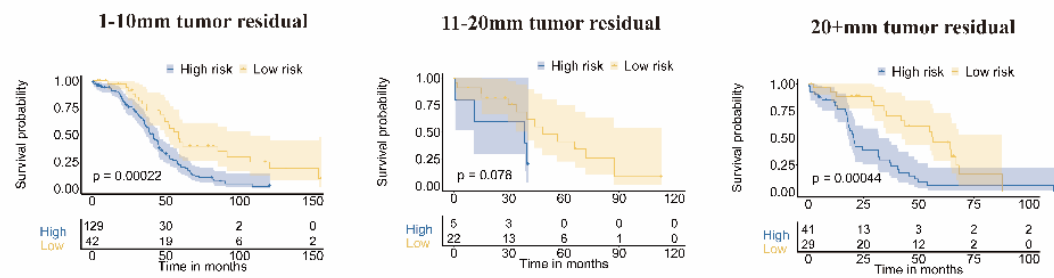

Figure 4. Stratification analysis to assess the prognostic value of risk score in subgroups divided based on age (A), FIGO stage (B), Grade (C), lymphatic invasion (D) and tumor residual size (E). 


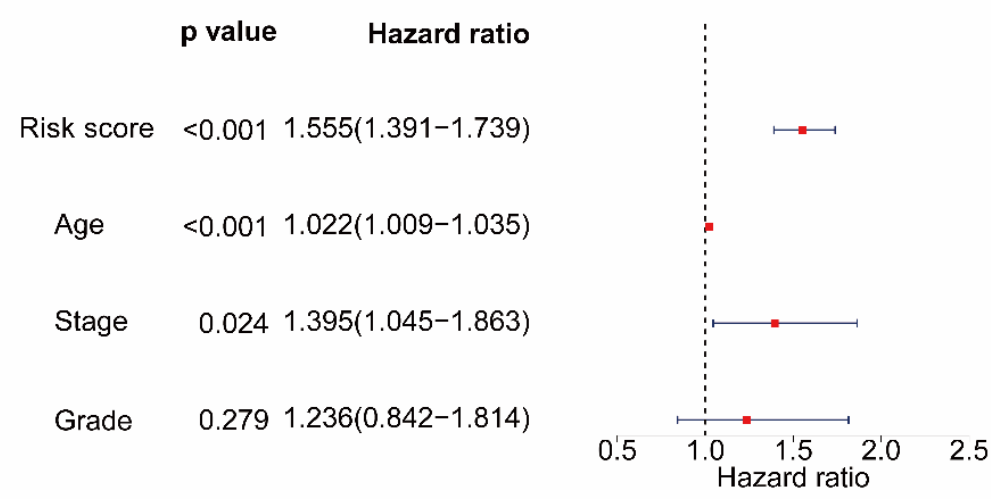

B Multivariate Cox regression analysis

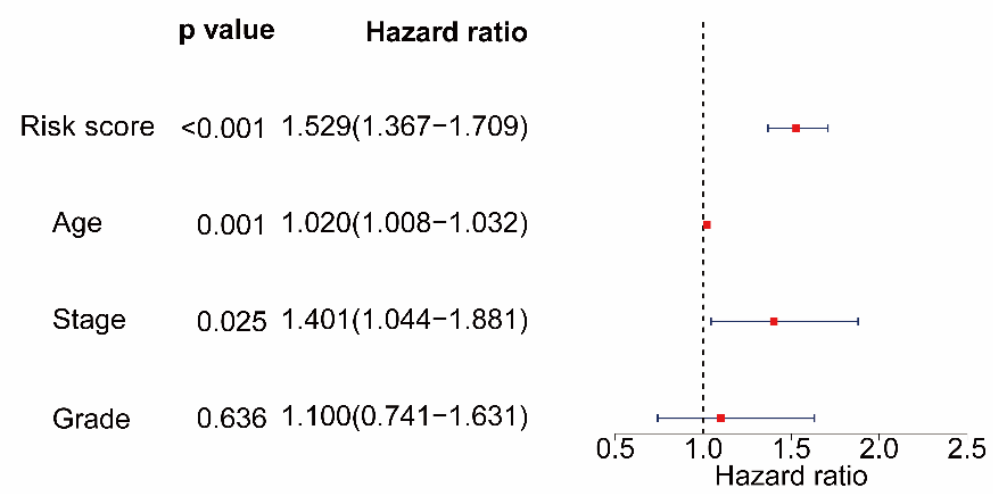

C

Points

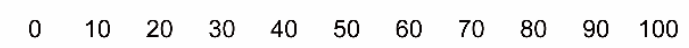

Age

Stage

\begin{tabular}{lllllll}
\hline 30 & 45 & 60 & 75 & 90 \\
\hline
\end{tabular}

Risk score

Total Points

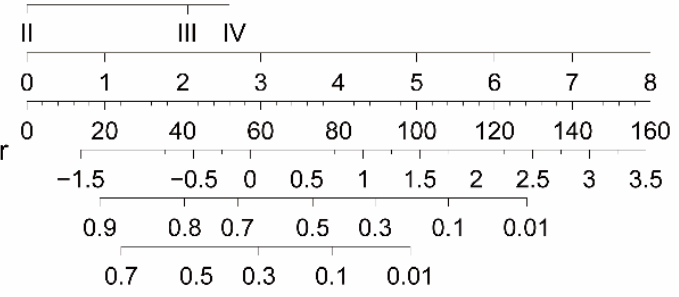

D

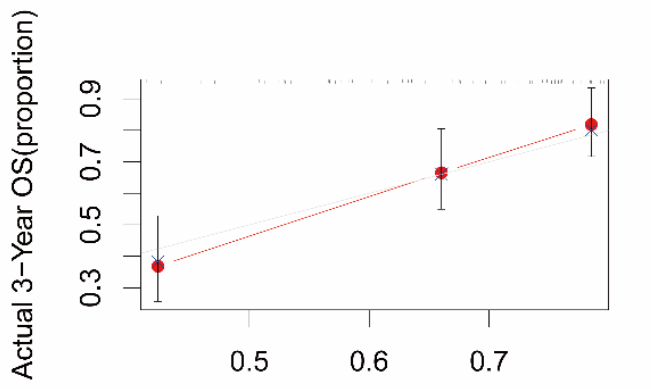

Nomogram-Predicted Probability of 3-Year OS

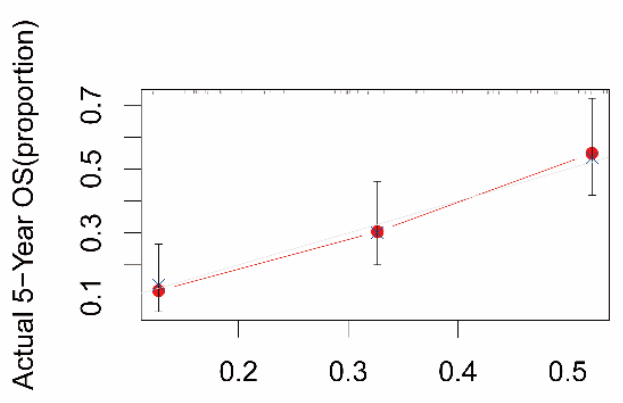

Nomogram-Predicted Probability of 5-Year OS

Figure 5. Construction of nomogram based on clinical feathers and risk score. (A-B)

The forest plot representing the univariate and multivariate analyses to select the 
independent prognostic predictors; (C) Establishment of a nomogram based on risk score, age, and stage to predict 3-, 5-year OS in the TCGA cohort; (D) Calibration plots of the nomogram to predict OS at 3-, 5-year.

A

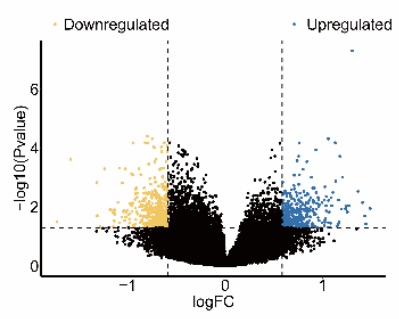

C
B

$\mathrm{D}$

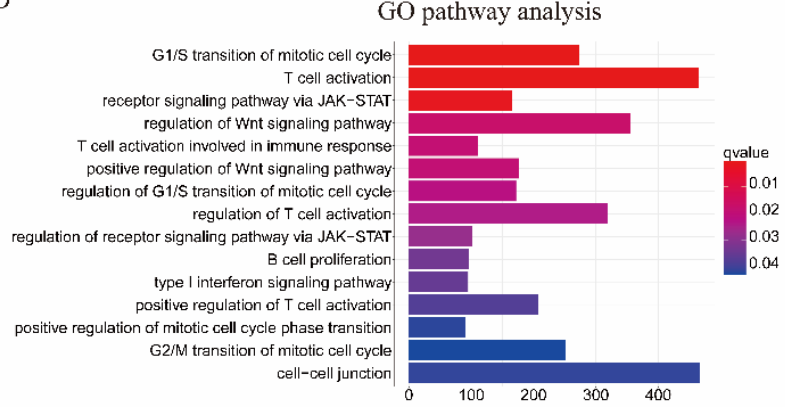

Figure 6. Functional analysis based on the DEGs between the two-risk groups in the TCGA cohort. (A) The volcano plot showed the different expression genes between high risk group and low risk group; (B) Analysis of Gene set enrichment analysis for DEGs; (C) The bubble plot displayed the analysis of KEGG pathway enrichment; (D) The bar plot revealed the analysis of GO pathway enrichment 
A

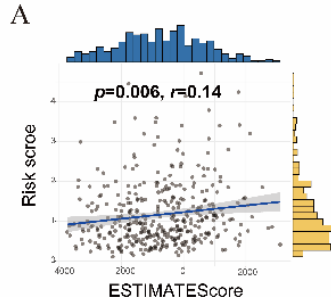

$\mathrm{C}$

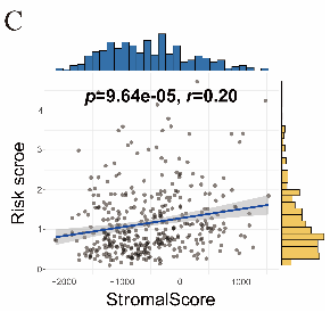

Stromalscore

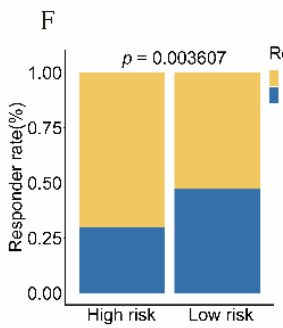

B

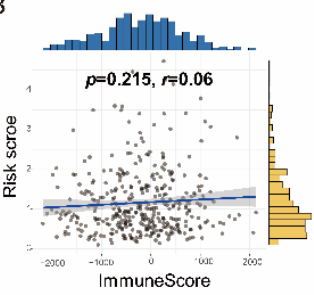

D

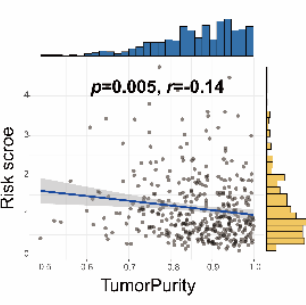

G

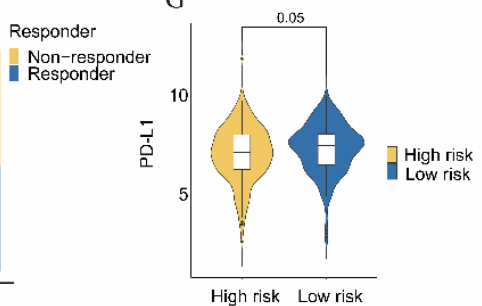

E

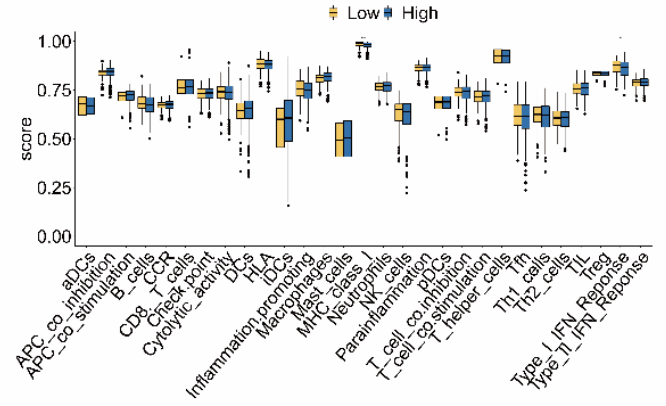

$\mathrm{H}$

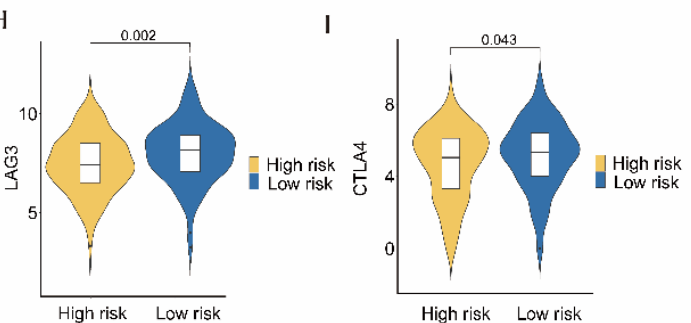

Figure 7. Association between the prognostic PRLs signature and immune microenvironment and immunotherapy response. (A-D) Correlation between risk score and microenvironment scores; (E) The differences of immune cells calculated by the ssGSEA analysis in high risk group and low risk group; (F) The proportion of the TIDE response to immunotherapy of patients with OC; (G-I) The different expression of immune checkpoints including PD-L1,CTAL4 and LAG3 in high and low risk groups. $* p<0.05,{ }^{* *} p<0.01,{ }^{* * *} p<0.001$. 


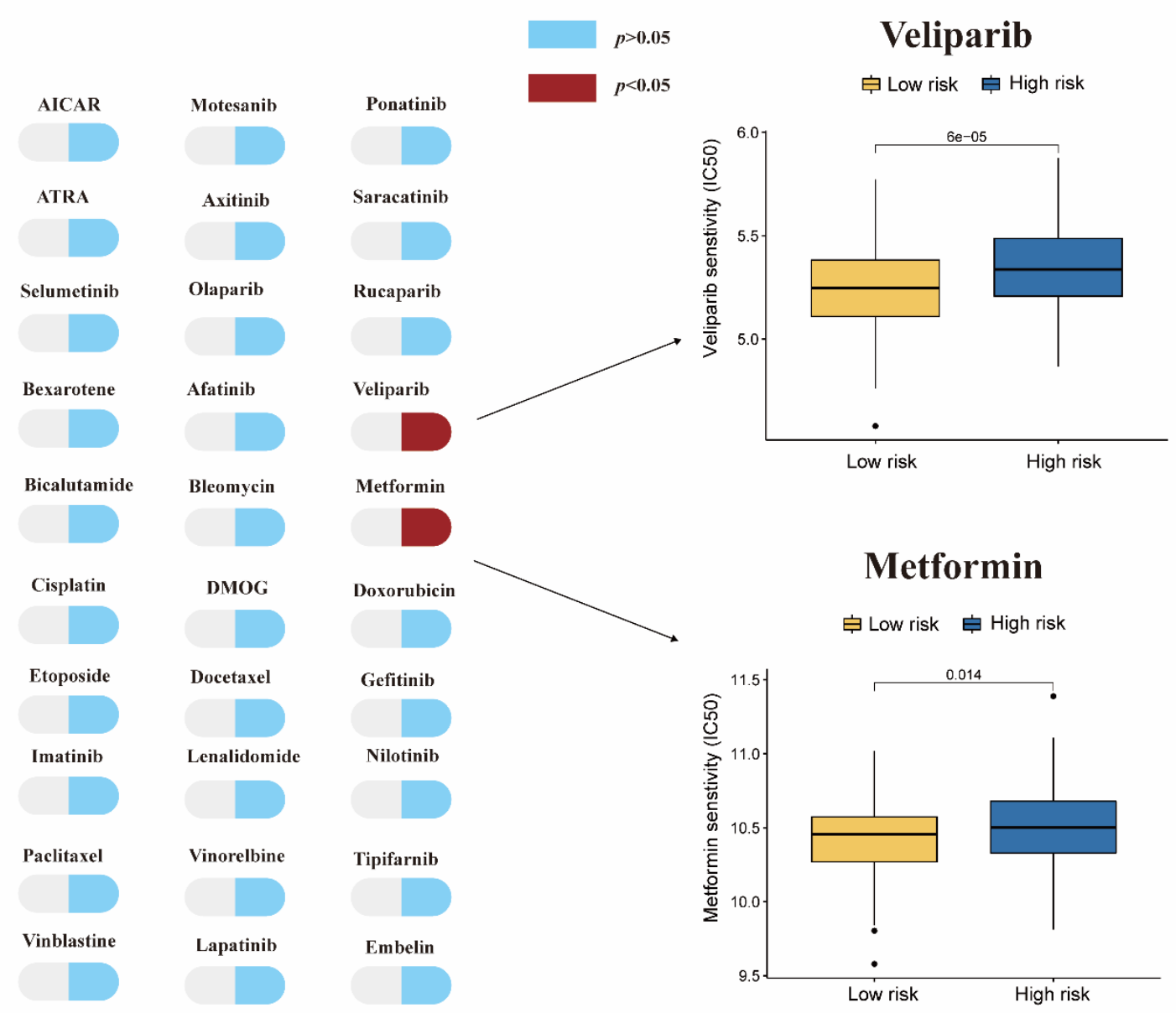

Figure 8. Estimated drug sensitivity in patients with high and low risk. 
A

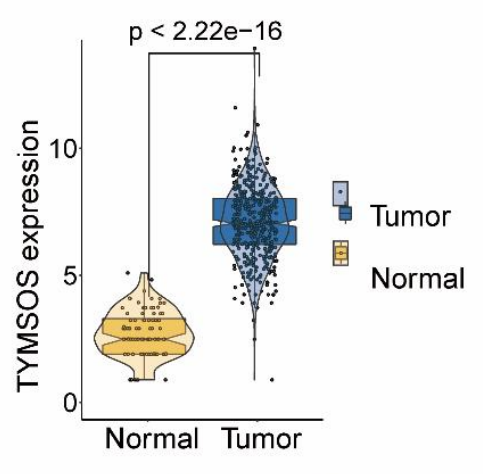

B

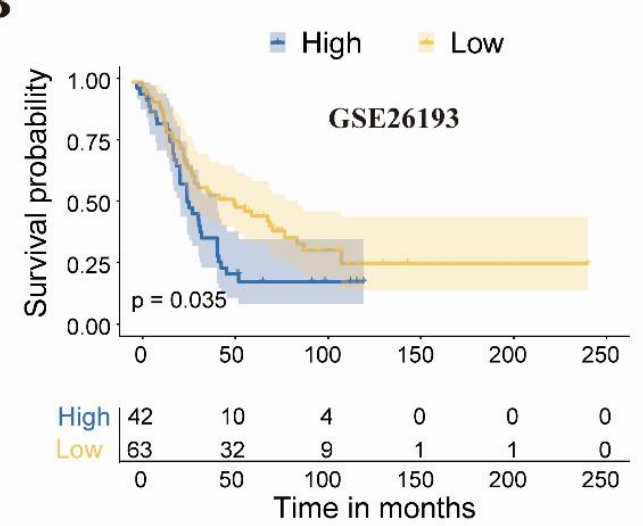

C

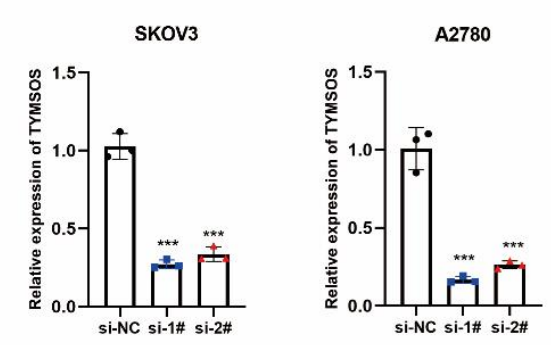

$\mathbf{F}$

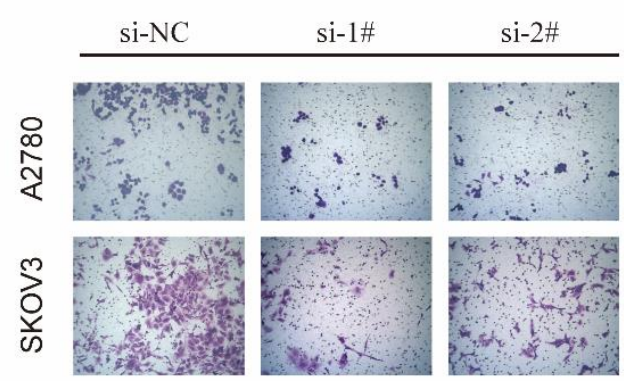

G

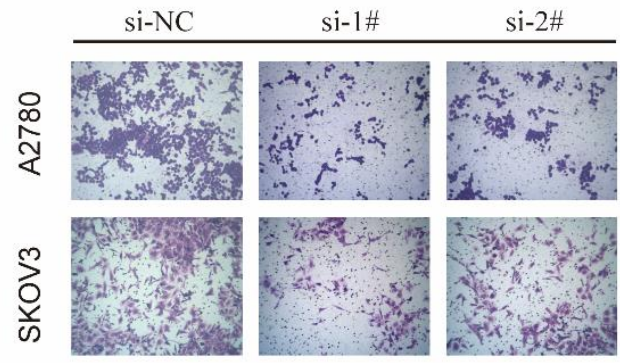

D
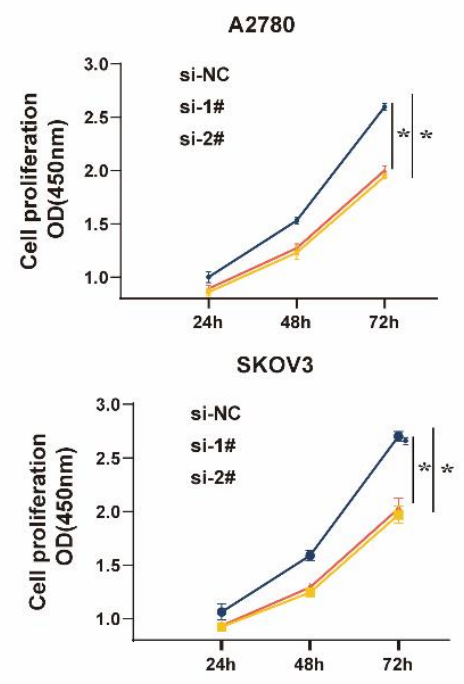

$\mathbf{E}$

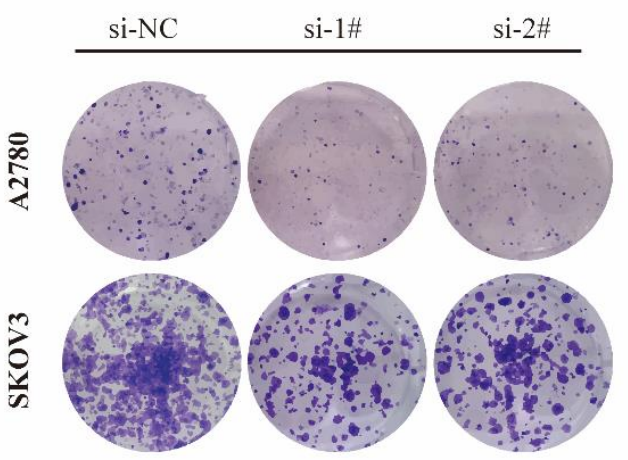

H

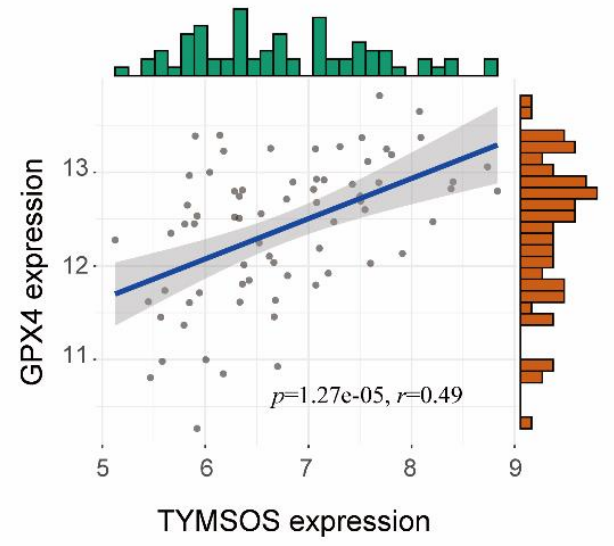

I

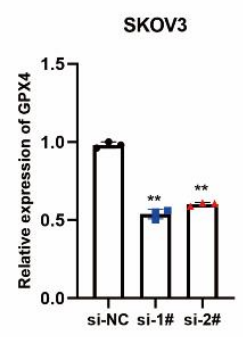

Figure 9. LncRNA TYMSOS promoted cell proliferation, invasion and migration via inhibiting pyroptosis. (A) TYMSOS was significantly upregulated in ovarian cancer 
tissues; (B) The KM plot showed that the high expression of TYMSOS had a remarkably worse prognosis in GSE26193 cohorts; (C) The expression of TYMSOS was significantly inhibited after treating with siRNA for 48h. (D-E) TYMSOS deletion significantly reduced the proliferation of A2780 and SKOV3 cells; (F-G) Inhibition of TYMSOS expression led to remarkable decrease in migratory capacity and invasion ability of A2780 and SKOV3 cells; (H) TYMSOS was positively correlated with GPX4 in the E-MTAB-1814 cohorts; (I) Inhibition of TYMSOS expression decreased the expression of GPX4 in A780 and SKOV3 cell lines. ${ }^{*} p<0.05,{ }^{* *} p<0.01,{ }^{* * *} p<0.001$. 
Table 1. Clinical characteristics of OC subjects $(n=377)$ in TCGA-RNA-seq dataset.

\begin{tabular}{|c|c|c|c|c|}
\hline Characteristics & Training cohort & Test cohort & Sum cohort & p-value* \\
\hline Age & & & & 0.63 \\
\hline Younger $(\leq 55 \mathrm{y})$ & 76 & 71 & 147 & \\
\hline Older $(>55 y)$ & 113 & 117 & 230 & \\
\hline FIGO Stage & & & & 0.20 \\
\hline I & 0 & 1 & 1 & \\
\hline II & 9 & 14 & 23 & \\
\hline III & 153 & 140 & 293 & \\
\hline IV & 26 & 31 & 57 & \\
\hline NA & 1 & 2 & 3 & \\
\hline Grade & & & & 0.13 \\
\hline G1 & 0 & 1 & 1 & \\
\hline G2 & 28 & 17 & 45 & \\
\hline G3 & 156 & 164 & 320 & \\
\hline G4 & 1 & 0 & 1 & \\
\hline GB & 1 & 1 & 2 & \\
\hline GX & 1 & 0 & 1 & \\
\hline NA & 0 & 2 & 2 & \\
\hline Tumor residual & & & & 0.42 \\
\hline No Macroscopic disease & 35 & 32 & 67 & \\
\hline $1-10 \mathrm{~mm}$ & 89 & 82 & 171 & \\
\hline $11-20 \mathrm{~mm}$ & 15 & 12 & 27 & \\
\hline$>20 \mathrm{~mm}$ & 29 & 41 & 70 & \\
\hline NA & 21 & 21 & 42 & \\
\hline Lymphatic invasion & & & & 0.32 \\
\hline YES & 53 & 48 & 101 & \\
\hline NO & 21 & 27 & 48 & \\
\hline NA & 115 & 113 & 228 & \\
\hline Subdivision & & & & 0.19 \\
\hline Left/ right & 57 & 45 & 102 & \\
\hline Bilateral & 123 & 132 & 255 & \\
\hline NA & 9 & 11 & 20 & \\
\hline Chemotherapy & & & & 0.32 \\
\hline Yes & 174 & 176 & 350 & \\
\hline Platinum-based & 163 & 169 & 332 & \\
\hline Others & 11 & 7 & 18 & \\
\hline NO & 15 & 12 & 27 & \\
\hline
\end{tabular}

* Training cohort vs Test cohort 


\section{Supplement materials}

Supplement Table 1. A list of Primer sequence used in the study.

Supplement Table 2. A list of all the PRLs screened by Pearson correlation analysis;

Supplement Table 3. The association between risk score and drug sensitivity of all the drugs;

Supplement Figure 1. The difference IC50 of cisplatin and paclitaxel between two groups. 